\title{
The sec6/8 Complex Is Located at Neurite Outgrowth and Axonal Synapse-Assembly Domains
}

\author{
Christopher D. Hazuka, Davide L. Foletti, Shu-Chan Hsu, Yun Kee, F. Woodward Hopf, and \\ Richard H. Scheller \\ Department of Molecular and Cellular Physiology, Howard Hughes Medical Institute, Stanford University School of \\ Medicine, Stanford, California 94305-5428
}

The molecules that specify domains on the neuronal plasma membrane for the delivery and accumulation of vesicles during neurite outgrowth and synapse formation are unknown. We investigated the role of the sec6/8 complex, a set of proteins that specifies vesicle targeting sites in yeast and epithelial cells, in neuronal membrane trafficking. This complex was found in layers of developing rat brain undergoing synaptogenesis. In cultured hippocampal neurons, the sec6/8 complex was present in regions of ongoing membrane addition: the tips of growing neurites, filopodia, and growth cones. In young axons, the sec6/8 complex was also confined to periodic domains of the plasma membrane. The distribution of synaptotagmin, synapsin1, sec6, and FM1-43 labeling in cultured neurons suggested that the plasma membrane localization of the sec6/8 complex preceded the arrival of synaptic markers and was downregulated in mature synapses. We propose that the sec6/8 complex specifies sites for targeting vesicles at domains of neurite outgrowth and potential active zones during synaptogenesis.

Key words: synaptogenesis; neurotransmission; secretion; exocytosis; synaptic vesicle; vesicle targeting
Neurons undergo a characteristic sequence of developmental events culminating in the formation of synapses. Initially, neurons polarize, elaborating functionally and morphologically distinct axons and dendrites (Dotti et al., 1988; Craig and Banker, 1994). This polarization arises, in part, through the specific intracellular trafficking of lipid and protein components, via vesicle intermediates, to appropriate locations in the cell (Calakos and Scheller, 1996). Vesicle trafficking is crucial for the membrane addition, which allows dynamic restructuring of axonal and dendritic plasma membrane in the process of neurite outgrowth. An important neurobiological question is how the vesicle-mediated membrane addition that underlies neurite outgrowth is accomplished. Studies of soluble $N$-ethylmaleimide-sensitive factor attachment protein receptors (SNAREs), a family of proteins which may negotiate vesicle and target membrane interactions, have provided much insight into the molecular mechanisms of secretion (Scheller, 1995; Sudhof, 1995). Vesicles are endowed with v-SNAREs of the vesicle-associated membrane protein (VAMP) family, which allow them to interact specifically with t-SNAREs of the syntaxin family located on the target membrane (Hay and Scheller, 1997). These interactions may be crucial mediators of membrane fusion events (Broadie et al., 1995; Hanson et al., 1997; Lin and Scheller, 1997; Weber et al., 1998). However, SNAREs of the syntaxin family are broadly localized along the

Received Oct. 15, 1998; revised Nov. 24, 1998; accepted Dec. 1, 1998.

We thank Drs. Svend Davanger and Erika Piedras-Renteria for assistance with hippocampal neuron cultures and experimental techniques; Drs. Susan McConnell and Alex Zhang for aid obtaining and analyzing brain section data; Dr. Charles F. Stevens for discussions; Dr. Jack Waters for help with FM1-43 experiments; Drs. Stephen J Smith, Cindy Adams, Jamie Jontes, and Susanne Ahmari for benefaction with confocal microscopy and discussion; and Drs. Cindy Adams, Richard Lin, Eva Ogielska, Rytis Prekeris, and Martin Steegmaier for critical reading of this manuscript.

Correspondence should be addressed to Dr. Richard H. Scheller at the above address.

Copyright (ㄷ) 1999 Society for Neuroscience $\quad 0270-6474 / 99 / 191324-11 \$ 05.00 / 0$ plasma membrane (see Fig. 3) and thus are probably not important for targeting of vesicles to specific domains (Galli et al., 1995). The molecules that define targeting domains for polarized constitutive secretion in neurons have yet to be described.

Targeting of vesicles to synaptic sites during development may use similar mechanisms as those involved in vesicle fusion underlying membrane outgrowth. Before contact with a postsynaptic target, axons possess mobile vesicle clusters bearing synaptotagmin, which fuse with the plasma membrane after stimulation (Matteoli et al., 1992; Kraszewski et al., 1995; Dai and Peng, 1996). Thus, growing axons must contain the molecular machinery required for constitutive exocytosis, endocytosis, and activitydependent vesicle release. However, it is unclear how vesicles become clustered at synapses. Although vesicle fusion in axons might occur anywhere along the plasma membrane, there must be membrane targets that signal the clustering of vesicles for synapse formation. Furthermore, it is unclear how sites of vesicle exocytosis are modified as the neuron forms stable contacts with postsynaptic partners.

One candidate for a vesicle targeting signal important in polarized exocytosis is the mammalian sec3/5/6/8/10/15/exo70/exo84 (sec6/8 or exocyst) complex. Supporting evidence comes from studies of the yeast Saccharomyces cerevisiae, mammalian epithelial cells (see Fig. 9), and rat brain. Most of the components of the yeast sec6/8 complex were initially discovered in a screen for secretory mutants in yeast and are homologs of members of the mammalian complex (Novick et al., 1980; Bowser and Novick, 1991; Bowser et al., 1992; Potenza et al., 1992; Ting et al., 1995; Hsu et al., 1996; TerBush et al., 1996; Guo et al., 1997; Hazuka et al., 1997; Kee et al., 1997). The yeast sec6/8 complex is associated with the plasma membrane and is highly concentrated at sites of active vesicle exocytosis: at the tip of a new growing bud (TerBush and Novick, 1995; Drubin and Nelson, 1996; TerBush et al., 1996; Finger and Novick, 1997; Finger et al., 1998) and just before 
cytokinesis at the neck of budding cells (Monde'sert et al., 1997). As in yeast, members of the mammalian sec6/8 complex associate to form a stable $17 \mathrm{~S}$ particle, which is present in rat brain (Hsu et al., 1996) and epithelial cells (Grindstaff et al., 1998). In polarized Madin-Darby canine kidney (MDCK) epithelial cells, antibodies directed against rat sec 8 block vesicle secretion at the basolateral membrane (Grindstaff et al., 1998). The sec6/8 complex is believed to act at the plasma membrane, upstream of the membrane fusion machinery, and is expressed in all tissues examined, suggesting an important role in constitutive vesicle targeting (Ting et al., 1995; Hazuka et al., 1997; Kee et al., 1997). These data are consistent with the hypothesis that the sec6/8 complex plays a role in the accumulation of vesicles at the plasma membrane, which is essential for polarized vesicle targeting.

We provide evidence that in addition to a role in the polarized exocytosis underlying neurite outgrowth, the sec6/8 complex is important for the formation of synapses. In slices of brain tissue and in cultured hippocampal neurons, the sec6/8 complex was highly expressed in regions undergoing neurite outgrowth and synaptogenesis. During synaptogenesis, expression of the sec6/8 complex along axons preceded the arrival of synaptic markers and then, later in development, colocalized with synapsin1 and FM143. However, in older neurons, sec6/8 complex localization at synapses was greatly reduced. These data point to roles for the sec6/8 complex in targeted vesicle secretion important for neurite outgrowth, as well as the development of synaptic release sites, and suggest that the complex is an early, transient marker for potential synapses.

\section{MATERIALS AND METHODS}

Brain sectioning and immunolabeling. Brains were removed from embryonic day 18 (E18) embryos, immediately submerged in $4 \%$ paraformaldehyde (PF) in PBS for $4 \mathrm{hr}$, and then transferred to $20 \%$ sucrose $-4 \%$ $\mathrm{PF}$ in PBS for $24 \mathrm{hr}$. Sixteen micrometer sections were cut using a cryostat, and sections were applied to Superfrost*/Plus slides (Fisher Scientific, Pittsburgh, PA). Adult and postnatal day 5 (P5) rats were perfused intracardially with $4 \% \mathrm{PF}$ in PBS. The brains were removed and submerged in $4 \% \mathrm{PF}$ in PBS for $1 \mathrm{hr}$, then transferred to $20 \%$ sucrose $-4 \%$ PF in PBS for $36 \mathrm{hr}$, and sectioned $(35 \mu \mathrm{m})$ on a freezing microtome. Sections from both fixation methods were then washed and blocked-permeabilized in $10 \mathrm{~mm}$ Tris, $30 \mathrm{~mm} \mathrm{NaCl}$ (TBS), $\mathrm{pH} 7.2$, containing $10 \%$ normal goat serum (NGS) and $0.3 \%$ Triton X-100 for 1 $\mathrm{hr}$. Primary antibodies were applied for $16 \mathrm{hr}$ at room temperature (RT) in TBS containing 5\% NGS and $0.3 \%$ Triton X-100. After washing in TBS, secondary antibodies were applied for $3 \mathrm{hr}$ at RT. Sections were then rinsed with TBS, placed on slides (adult and P5 sections), placed under coverslips, and visualized with a scanning laser confocal microscope designed by Drs. Stephen J Smith and Noam Ziv (Stanford University, Stanford, CA).

Preparation of hippocampal neuron cultures. Embryonic hippocampal cell cultures were prepared from the hippocampi of 18- to 19-d-old fetal rats as described previously (Banker and Cowan, 1977; Fletcher et al., 1991). Hippocampi were treated with $0.05 \%$ trypsin and $0.53 \mathrm{~mm}$ EDTA for $15 \mathrm{~min}$ at $37^{\circ} \mathrm{C}$, and cells were dissociated by triturating with a silicon-coated fire-polished pipette. Dissociated cells were plated at $\sim 5000$ cells $/ \mathrm{cm}^{2}$ in neuronal medium [Earle's MEM containing: glucose $(6 \mathrm{mg} / \mathrm{ml})$, apo-transferrin $(100 \mu \mathrm{g} / \mathrm{ml})$, ovalbumin $(0.1 \%)$, NaPyruvate $(1 \mathrm{mM})$, putrescine $(100 \mu \mathrm{M})$, insulin $(5 \mu \mathrm{g} / \mathrm{ml})$, progesterone $(20 \mathrm{nM})$, and selenium dioxide $(30 \mathrm{nM})$ ] supplemented with $10 \%$ equine serum (HyClone, Logan, UT). These cells were then allowed to attach to poly-L-lysine-coated coverslips for 2-6 hr, after which the coverslips were placed in cultures of previously prepared astroglia in neuronal medium. Neurons were fed once every $7 \mathrm{~d}$. In some experiments, cultures were prepared from the hippocampi of 3-d-old postnatal rats as described previously (Malgaroli et al., 1995).

Immunocytochemistry of cultured cells. Cells were fixed by one of three methods. Some cultures were submerged in $4 \% \mathrm{PF}$ and $120 \mathrm{~mm}$ sucrose in PBS for $20 \mathrm{~min}$, extracted in $-20^{\circ} \mathrm{C}$ methanol for $6 \mathrm{~min}$, dehydrated, and rehydrated in $0.1 \mathrm{M}$ glycine in PBS. In some experiments, the PF or methanol step was omitted. The best method of fixation varied with the antibody used and was adjusted appropriately. Cells were probed with a variety of antibodies diluted in PBS containing $0.4 \%$ saponin- $2 \%$ NGS and $1 \%$ bovine serum albumin in PBS. The 9H5 monoclonal antibody was used for sec6 labeling (Kee et al., 1997). The 2E12 monoclonal antibody was used for sec8 labeling (Hsu et al., 1998). An affinity purified rabbit polyclonal antibody directed against bovine brain synapsin1 (1a and $1 \mathrm{~b}$ ) was purchased from Chemicon (Temecula, CA). A rabbit polyclonal antibody raised against bovine brain MAP2 was purchased from Biogenesis (Sandown, NH). The rabbit polyclonal antibody to synaptotagmin was made by Ken Miller (Stanford University) and was described in Jacobsson et al. (1994). After incubation with primary antibodies for either $1 \mathrm{hr}$ at RT or overnight at $4^{\circ} \mathrm{C}$, the cells were rinsed three times for $5 \mathrm{~min}$ in PBS and incubated with secondary antibodies for $1 \mathrm{hr}$ at RT. Secondary antibodies were purchased from Jackson ImmunoResearch (West Grove, PA) and included dichlorotriazinyl amino fluorescein (DTAF)-conjugated AffiniPure goat anti-mouse $\mathrm{IgG}$, Texas Redconjugated AffiniPure donkey anti-rabbit IgG, and Cy5-conjugated AffiniPure goat anti-mouse IgG. Finally, cells were washed three times for 5 min, mounted onto slides, and visualized using either a Zeiss (Oberkochen, Germany) Axiophot or scanning laser confocal microscope.

Functional labeling of presynaptic axon terminals with FM1-43 and retrospective immunocytochemistry. FM1-43 loading was performed essentially as described by Ryan and Smith (1995). Coverslips with 8-9 days in vitro (div) embryonic hippocampal neurons were mounted in microscope chambers using vacuum grease and fitted with electrodes. After mounting onto the microscope, cultured neurons were constantly superfused at $34^{\circ} \mathrm{C}$ with a solution of $119 \mathrm{~mm} \mathrm{NaCl}, 2.5 \mathrm{~mm} \mathrm{KCl}, 2 \mathrm{mM} \mathrm{CaCl}_{2}$, $2 \mathrm{mM} \mathrm{MgCl}_{2}$, and $25 \mathrm{mM} \mathrm{HEPES}, \mathrm{pH} 7.4$, containing $50 \mu \mathrm{M} \mathrm{D}-2$-amino5-phosphonovaleric acid and $10 \mu \mathrm{M}$ 6-cyano-7-nitroquinoxaline-2,3dione. Neurons were stimulated in the presence of $15 \mu \mathrm{M}$ FM1-43 (Molecular Probes, Eugene, Oregon) with 300 field pulses of $30 \mathrm{~mA}$ at 10 $\mathrm{Hz}$ and then allowed to endocytose the dye for $30 \mathrm{sec}$. After 15-20 min of washing with dye-free superfusion solution, confocal images were obtained using custom multisite software (Drs. Noam Ziv and Stephen J Smith). FM1-43 was then unloaded from vesicles by stimulation in the absence of the dye. After unloading, the neurons were imaged again and then immediately fixed and labeled as described above. The same fields were then imaged for immunofluorescence and compared with the signal collected from the FM1-43 images.

Quantitative analyses methods. Cultures of different ages labeled with antibodies to synapsin 1 and $\sec 6$ were used for quantification of the colocalization of sec6 and synapsin1. Synapsin1-positive sites were counted and scored for the presence of sec 6 immunofluorescence by visualizing 10-20 fields containing cell bodies at each age through the $40 \times$ objective of a Zeiss Axiophot microscope. At least 1000 synapsin 1 sites were counted for each age. Each field was quantified and averaged as shown in Figure 7. Alternatively, confocal images were obtained at the same days in vitro and $>15$ fields were analyzed at each age for colocalization using the Metamorph program (Universal Imaging Corporation, West Chester, PA). The Metamorph colocalization program is expected to give conservative estimates of colocalization for two reasons. (1) Maximal levels will be less because the sec6/8 complex and synapsin 1 do not overlap exactly (see Figs. 4, 5); thus, the colocalization will never be $100 \%$. (2) The Metamorph program will include dendritic contributions to sec6 immunofluorescence (see Fig. $6 L$ ); thus, at later ages, the colocalization will be greater. These two predictions were borne out in Figure 7 in which the colocalization decreases from $\sim 80$ to $40 \%$. Nevertheless, this unbiased automated method substantiated the results obtained by counting, thus confirming the trend of less colocalization with age. The time course of decrease of colocalization with age was similar using the two quantification techniques; both showed a half-maximal colocalization at $\sim 7-8$ div.

Colocalization analysis of FM1-43, synapsin1, and sec6 fluorescence was also performed using the Metamorph program and is presented in Tables 1, 2. A total of 300 sec6 sites and 80 FM1-43 sites, which both loaded and unloaded, were counted.

A histogram of the frequency distribution of distances between sec6/8 puncta was accomplished using the VIEW program (written by Drs. Noam Ziv and Stephen J Smith) (data not shown). The average distance was calculated from this distribution.

Reagents. Unless otherwise stated, all reagents were obtained from Sigma (St. Louis, MO) or Life Technologies (Grand Island, NY). 
Table 1. Quantification of colocalization of sec6, synapsin1, and FM143 fluorescence in stimulated synapses

\begin{tabular}{llll} 
sec6 & synapsin1 & FM1-43 & $\%$ of sec6 sites \\
\hline+ & - & - & $66 \pm 9$ \\
+ & + & - & $14 \pm 9$ \\
+ & + & + & $16 \pm 6$ \\
+ & - & + & $4 \pm 5$
\end{tabular}

sec6 sites were counted and scored for the presence or absence of synapsin 1 and/or FM1-43. Percentages were obtained by dividing the number of each group by the total number of sec6 sites.

Table 2. Quantification of colocalization of sec6, synapsin1, and FM143 fluorescence in stimulated synapses

\begin{tabular}{lll} 
synapsin1/FM1-43 & sec6 & $\%$ of synapsin1/FM1-43 sites \\
\hline+ & + & $33 \pm 14$ \\
+ & - & $67 \pm 14$
\end{tabular}

Sites containing both FM1-43 and synapsin1 were counted and scored for the presence or absence of sec6. Percentages were obtained by dividing the number of each group by the total number of FM1-43/synapsin1 sites.

\section{RESULTS}

\section{sec6 expression is abundant in regions of the cerebral cortex undergoing synaptogenesis}

To determine whether the sec6/8 complex plays a role in polarized neurite outgrowth and/or synaptogenesis during development of the cerebral cortex, brains from rats of different ages were sectioned and labeled with antibodies to sec6, synaptotagmin, and/or synapsin1. At all ages, similar results were obtained with synaptotagmin and synapsin1 (data not shown). Because sec6 and sec8 are stable components of the sec6/8 complex (Hsu et al., 1996), either sec6 or sec 8 antibodies were used to analyze its distribution. In fetal brains, synaptogenesis is confined to regions of the developing cortex, termed the subplate, and the marginal layer (Chun and Shatz, 1988, 1989). In sections of brain from E18 rats, high levels of sec6 immunoreactivity were restricted to the subplate layer and the marginal layer (Fig. $1 A$ ). These two layers were also specifically labeled by antibodies to synaptotagmin (Fig. 1B).

Soon after birth, synaptogenesis occurs throughout the cortex. Indeed, synaptotagmin and sec6 immunofluorescence were broadly distributed throughout the cortex of P5 rat brains (Fig. $1 D, E)$. Although the intensity of synaptotagmin labeling is low at P5, it is clearly above an IgG background, which has been subtracted from the images shown. The correlated change in sec6 and synaptotagmin labeling from E18 to P5 suggests that the sec6/8 complex is located at sites of developing synapses. Interestingly, the intensity of sec6 labeling was greatly decreased in adult compared with P5 animals (Fig. $1 E, G$ ) whereas the intensity of synaptotagmin labeling was increased (Fig. 1D,F), suggesting that the sec6/8 complex may either be greatly reduced in its abundance and therefore not required for mature neuronal function or modified and not recognized by the antibody. The presence of high levels of sec6/8 complex in brain regions undergoing synaptic development suggests a role for the sec6/8 complex in neurite outgrowth and synaptogenesis.

\section{The sec6/8 complex is located in the terminals of} growing neurites and in periodic domains of axons

The subcellular distribution of the sec6/8 complex in neurons was analyzed to determine whether it plays a role in the development

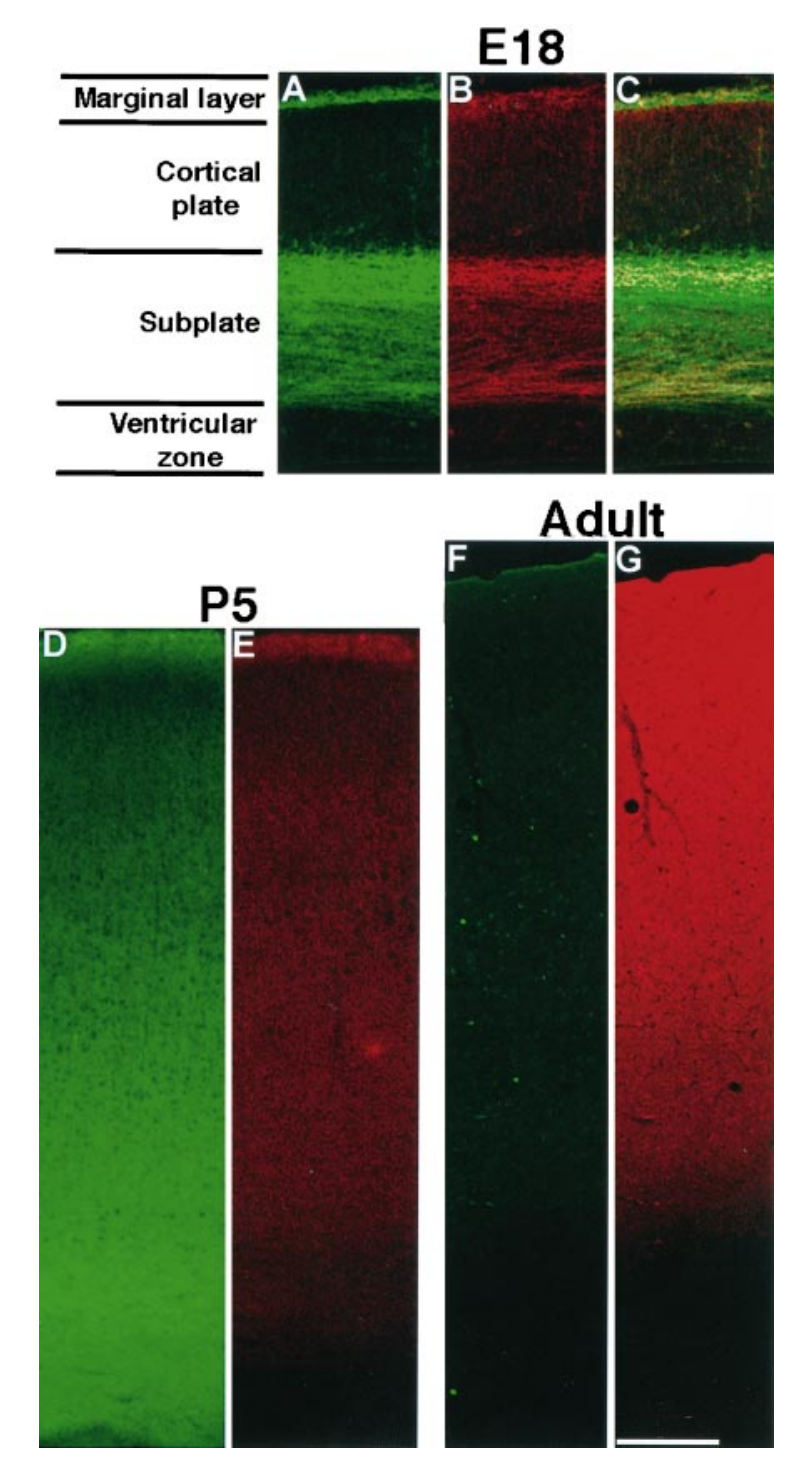

Figure 1. sec6 is highly expressed at sites of synaptogenesis during cortical development. Sections of E18 brains were labeled with antibodies directed against sec6 $(A)$ and synaptotagmin $(B)$. $C$, Overlay of images in $A$ and $B$. Both antibodies strongly labeled the cortical subplate and marginal layers. Sections of $\mathrm{P} 5(D, E)$ and adult $(F, G)$ brain were labeled with antibodies directed against $\sec 6(D, F)$ and synaptotagmin $(E, G)$. In all micrographs, the pia is up, and the ventricle is down. Scale bar, $220 \mu \mathrm{m}$.

of neuronal polarity and/or synaptic structures. Primary cultures of embryonic hippocampal neurons provide a useful system in which to study these phenomena. These cells exhibit polarized axonal and dendritic domains regardless of contact with other cells and can be grown separately from glia, making visualization of fine processes possible (Banker and Cowan, 1977; Fletcher et al., 1991). Furthermore, as neurons mature and contact each other, they develop functional synapses (Bartlett and Banker, 1984). Neurons at various stages of development were decorated with antibodies directed against sec6 and sec8 to discern where the sec6/8 complex was distributed with respect to active vesicle exocytosis.

At 2 div, neurons that have not yet formed synapses (Fletcher et al., 1991) exhibited intense sec6 labeling of the cell body, dendritic growth cones, axonal branch points, and axonal growth cones (Fig. $2 A, B$ ). Labeling of the cell body probably represented 

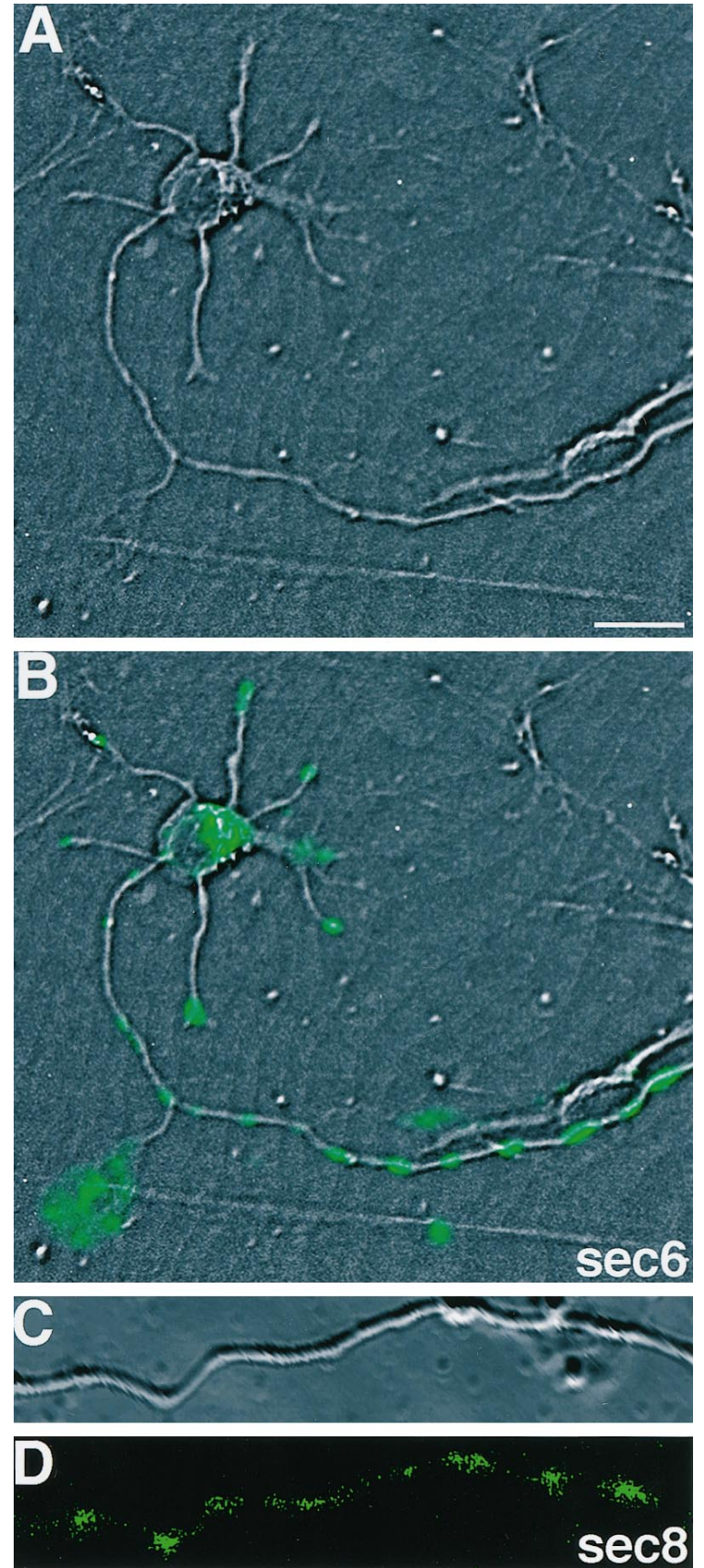

Figure 2. The sec6/8 complex is present in growth cones and at regular intervals along axons. Neurons in early stages of development in culture were labeled with antibodies to sec 6 and sec 8 . $A$, Phase-contrast image of a 2 div neuron. $B$, sec6 immunofluorescence of the same neuron overlaid on the phase-contrast image. sec6 immunofluorescence was found in a discrete localization both at the growth cones of dendrites and axons, and in periodic domains along the axon. $C, D$, Antibodies directed against the sec8 subunit of the complex also labeled axons in a periodic pattern. $C$, Nomarski image. $D$, Immunofluorescence image. Scale bar: $A, B, 10 \mu \mathrm{m}$; $C, D, 5 \mu \mathrm{m}$.

a soluble pool of the complex before its targeting to the plasma membrane. The six shorter processes with sec6 at their terminal growth cones in Figure $2 B$ are likely immature dendrites (Dotti and Banker, 1987). This pattern of sec6/8 complex labeling is consistent with a role in targeting of vesicles to sites of exocytosis in developing neurons, such as growth cones.
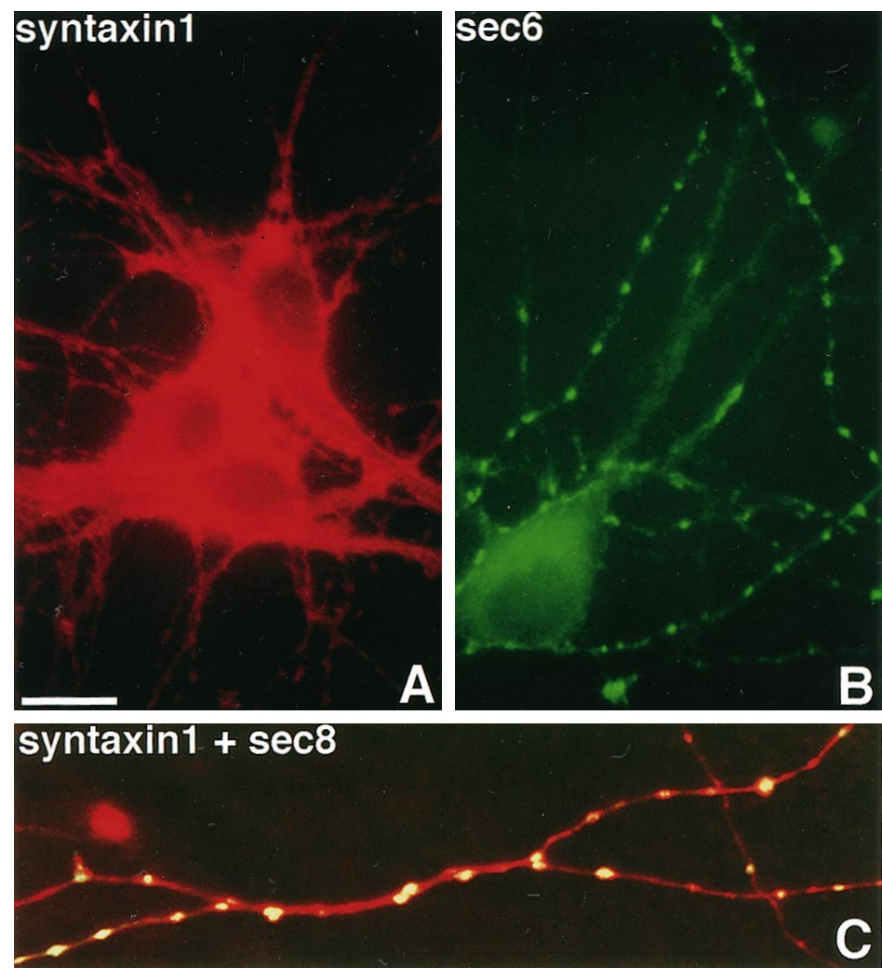

Figure 3. The sec6/8 complex is localized at discrete axonal domains. The distribution of the sec6/8 complex in axons was compared with that of the t-SNARE syntaxin1 in 7 div neurons. Syntaxin 1 (A, red; $C$, red and yellow) is found continuously throughout the axonal plasma membrane, whereas sec6 $(B)$ and $\sec 8(C$, yellow) are present in distinct periodic regions. This labeling pattern is observed in neurons fixed with both methanol $(A, B)$ and paraformaldehyde $(C)$ and with two different antibodies to two different members of the sec6/8 complex. Scale bar: $A$, $B, 7 \mu \mathrm{m} ; C, 4 \mu \mathrm{m}$.

Interestingly, sec6 (Fig. 2B) and sec8 immunofluorescence (Fig. 2D) were observed along axons in a periodic pattern, indicating that the sec6/8 complex was located in discrete domains along the axon. The average distance between these domains was $3.2 \pm 1.1 \mu \mathrm{m}$. Labeling with antibodies to syntaxin1 (Fig. 3A,C), $\beta$-catenin (data not shown), and the septin CDC10 protein (Hsu et al., 1998) using identical fixation and immunocytochemical techniques did not reveal a similar pattern of periodic staining, ruling out the possibility of fixation and/or labeling artifacts. These domains were further characterized by double labeling with antibodies to sec6 and synaptotagmin, a vesicle marker. Fields containing single axons were selected and visualized at high magnification. The sec6 staining was located peripherally in a "shell" around the vesicle marker (Fig. 4A,B), suggesting that the periodic discrete domains of sec 6 were present only on the plasma membrane. This plasma membrane labeling is consistent with previous biochemical experiments, which demonstrated the existence of a pool of the sec6/8 complex bound to the plasma membrane (Hsu et al., 1996). Filopodia originating from both the growth cone and the axon also contained punctate sec6 immunofluorescence (Fig. $4 B-D$ ). All areas of sec6/8 complex labeling are likely locations of membrane trafficking activity in growing axons.

Older cultures (6-9 div) were double-labeled with antibodies to MAP2 (a dendritic marker) and sec6 to determine whether the sec6/8 complex is present in dendrites, as well as axons. Although some lighter, diff use sec6 labeling was observed in dendrites and 

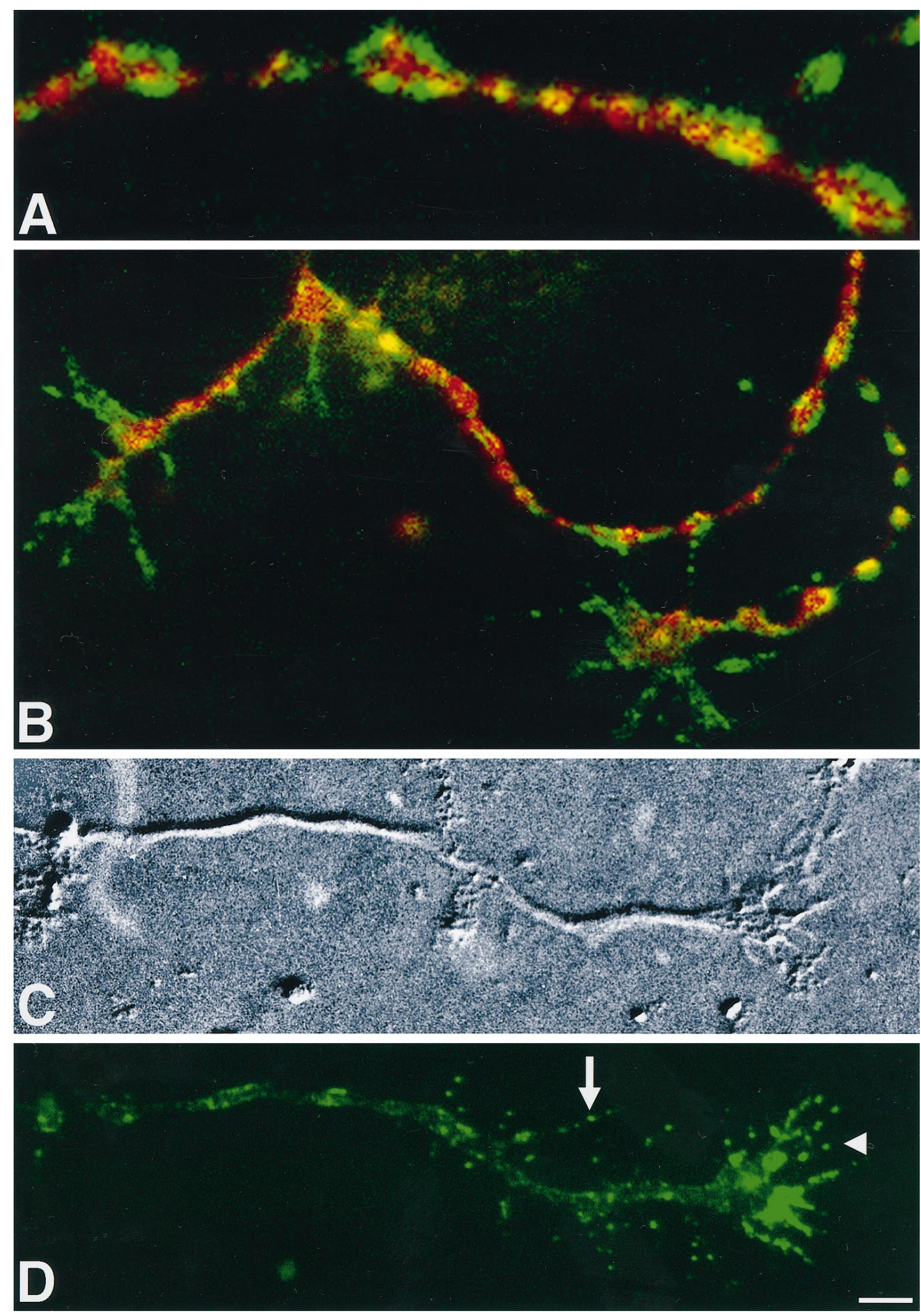

Figure 4. The sec6/8 complex is present along the axonal membrane at discrete domains and in filopodia. Distribution of sec6 (green) and synaptotagmin (red) at the distal end of growing axons. $A, B$, Terminal of a growing axon and growth cone in a postnatal culture. $C$, Nomarski image of an axon with a growth cone in an embryonic culture. $D$, Immunofluorescence image of the axon shown in $C$; sec6 was found in a punctate distribution in filopodia along the axon (arrow) and in the growth cone (arrowhead), in addition to its characteristic periodic pattern along the axonal membrane. In both culture types, sec6 appeared predominantly at the plasma membrane along the axon. Scale bar: $A, 2.7 \mu \mathrm{m} ; B-D, 5.4 \mu \mathrm{m}$. 

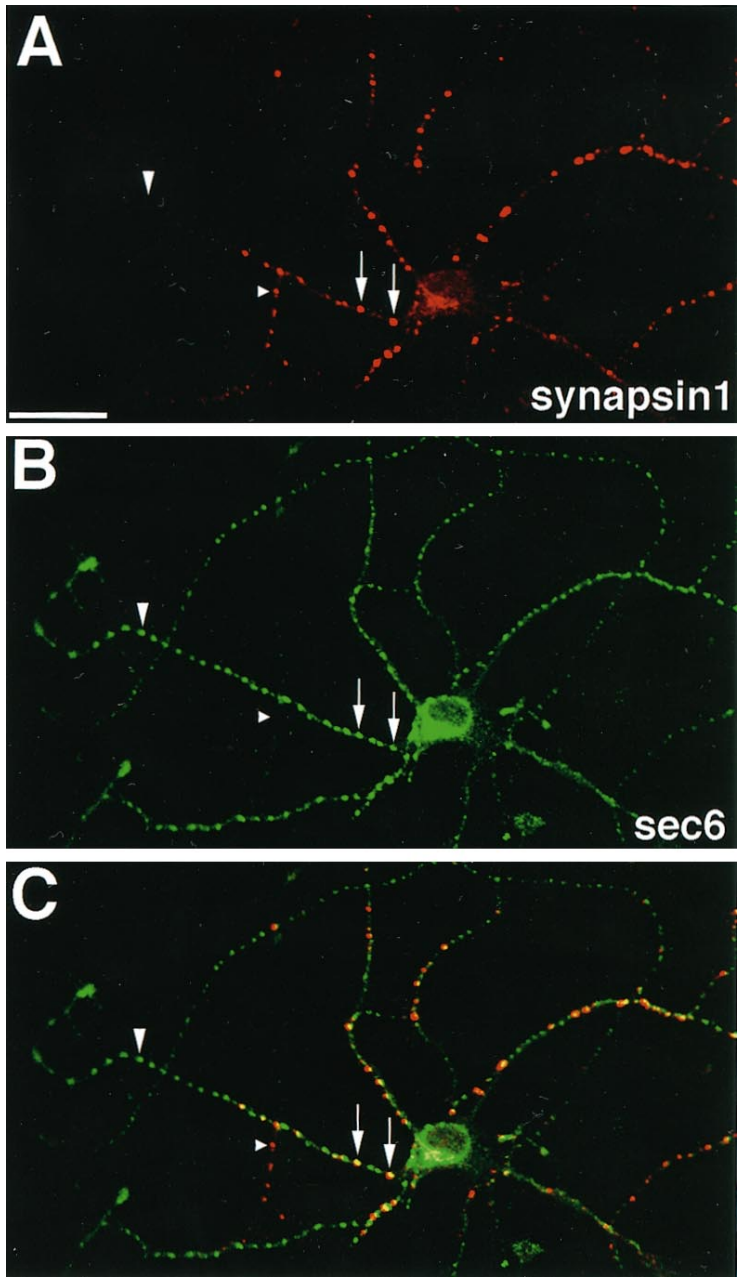

Figure 5. Synapsin 1 is precisely apposed to sec6/8 complex sites in axons. $A$, Labeling of a 6 div neuron with antibodies to synapsin1. $B$, Same neuron labeled with antibodies to rat sec6. $C$, Overlay of $A$ and $B$. This neuron had likely established many mature synapses and was still forming new ones. Synapsin1 labeling can be observed in both the absence (small arrowhead) and presence (arrows) of sec6 labeling. sec6 labeling can be seen in the absence of synapsin1 staining (large arrowhead). Scale bar, $25 \mu \mathrm{m}$.

the cell body (Figs. 5, 6H), more intense sec6 staining was found predominantly in MAP2-negative axons (data not shown). The diffuse dendritic and cell body labeling could represent the soluble pool reported previously (Hsu et al., 1996).

\section{The sec6/8 complex marks sites along axons that can become synapses}

The discrete labeling pattern of the sec6/8 complex in the axons of very young neurons suggests that it may be a molecular spatial landmark for vesicle delivery to nascent presynaptic specializations. To investigate this idea, cultures were labeled with antibodies directed against sec 6 and synapsin 1 at 6 div, a time when many initial contacts are forming between presynaptic and postsynaptic neurons (Fletcher et al., 1991). We used the presence of focal points of synapsin1 immunofluorescence as a marker for synapses (Bartlett and Banker, 1984; Fletcher et al., 1991; Ryan et al., 1993). Many axons were found growing around cell bodies and dendrites, likely establishing the initial stages of synapse formation (Fig. $5 A-C$ ). sec6 was distributed in a periodic manner in these axons (Fig. $5 B$ ). In axonal regions labeled by sec6 antibod- ies, many, but not all, of the sec6 domains also contained synapsin1 immunofluorescence (Fig. $5 A, C$ ). In these regions, synapsin1 labeling was not observed in between, but only coincident with, the periodic sec6 labeling along axons, suggesting that sec6/8 complex sites are synaptic precursors.

Unexpectedly, many axonal regions containing synapsin1immunoreactive synapses did not contain sec6 labeling. These regions likely contain mature synapses devoid of the sec6/8 complex because they are observed apposed to dendrites (Nomarski image not shown). Synapsin1-positive sec6-negative regions were observed along entire axons (Fig. $6 L$ ), suggesting that the axonal regions imaged were in different developmental stages. The observations that, in younger axonal processes ( 3 div), synapsin 1 was not found in the absence of sec6 and that a high number of sec6 labeled axons were present indicate that synapsin1 does not accumulate in the absence of sec6 and that sec6 accumulation precedes that of synapsin1.

To investigate the relationship between sec6/8 complex labeled axonal domains and mature synapses, we studied the time course of sec6/synapsin 1 coexpression in neuronal cultures of different ages. In young cultures ( $4 \mathrm{div}$ ), many axons were found growing around cell bodies and dendrites (Fig. 6A,E,I). sec6 was distributed in the typical periodic manner in these axons. Although a small percentage of the sec6 labeled axonal domains contained faint synapsin1 staining, the sites of axodendritic contact contained intense synapsin1 labeling (Nomarski image not shown). At older stages of development (6-12 div), more synapsin1 sites were observed (Fig. 6B-D), with decreased colocalization with sec6 (Fig. $6 J-L$ ). At 12 div, many synapsin1 labeled sites without sec6 staining were present in axons that run along diffusely sec6-labeled dendrites (Fig. 6L). Some axons at 12 div still contained labeling of sec6 but not of synapsin1 (Fig. 6L, left), likely representing axons that had not yet contacted dendrites. These results suggest that the sec6/8 complex is present in the presynaptic component of newly formed, but not mature, synapses.

To more rigorously determine the relationship between synap$\sin 1$ and sec6, immunofluorescence was quantified at different developmental ages. A large number of fields (see Materials and Methods) were imaged using either conventional or scanning laser confocal microscopy in each of several different labeled preparations of hippocampal cultures. Synapsin1-immunoreactive sites were counted at low magnification and scored for the presence or absence of sec 6 immunofluorescence. Colocalization decreased from $\sim 100$ to $20 \%$ between 3 and 12 div (Fig. 7, solid line). Similar results were observed using a less precise, automated method (Fig. 7, dashed line) (see Materials and Methods for details). Colocalization of the sec 6 immunoreactivity in synapsin 1 sites remained at $\sim 20 \%$ later in culture because axons were continually growing in embryonic hippocampal cultures and synaptogenesis occurs at both early times and later ages (Fletcher et al., 1994). These data support the hypothesis that the sec6/8 complex tags regions of the plasma membrane for potential delivery of synaptic vesicles but is downregulated in mature synapses.

\section{The sec6/8 complex is not required for functional local cycling of synaptic vesicles}

Mature synapses are characterized by specialized local vesicle cycling in the presynaptic region, where vesicles are repeatedly exocytosed and endocytosed (Ryan and Smith, 1995). To test whether synapsin1 and/or sec6 sites support functional vesicle cycling, we monitored vesicle recycling with the vital dye FM1-43 (Ryan and Smith, 1995; Betz et al., 1996). Cultures, aged 8-9 div 


\section{synapsin1}
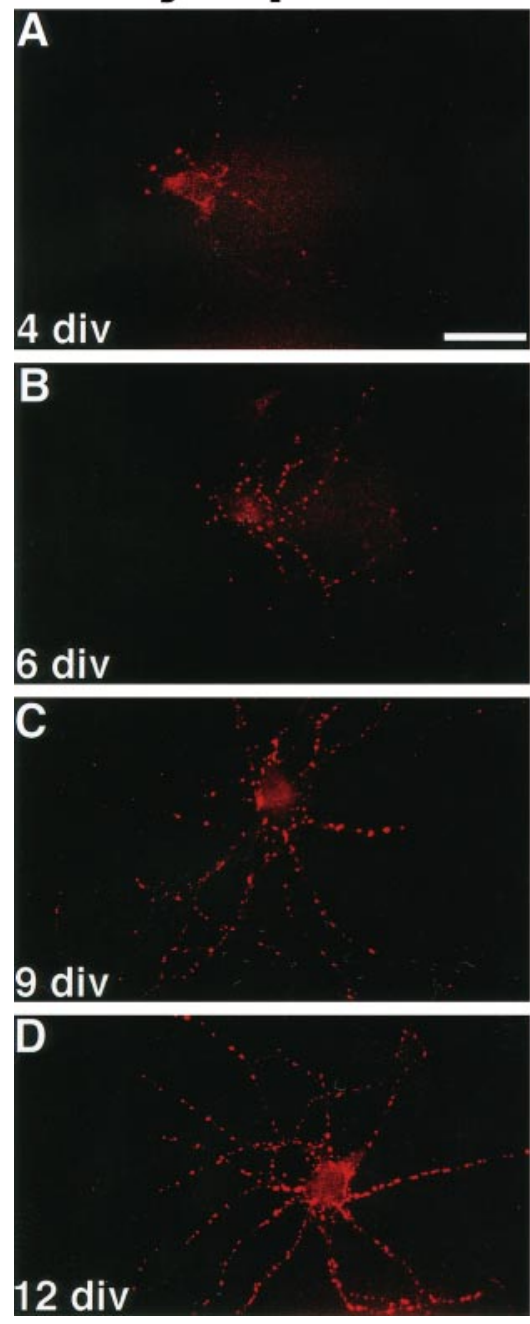
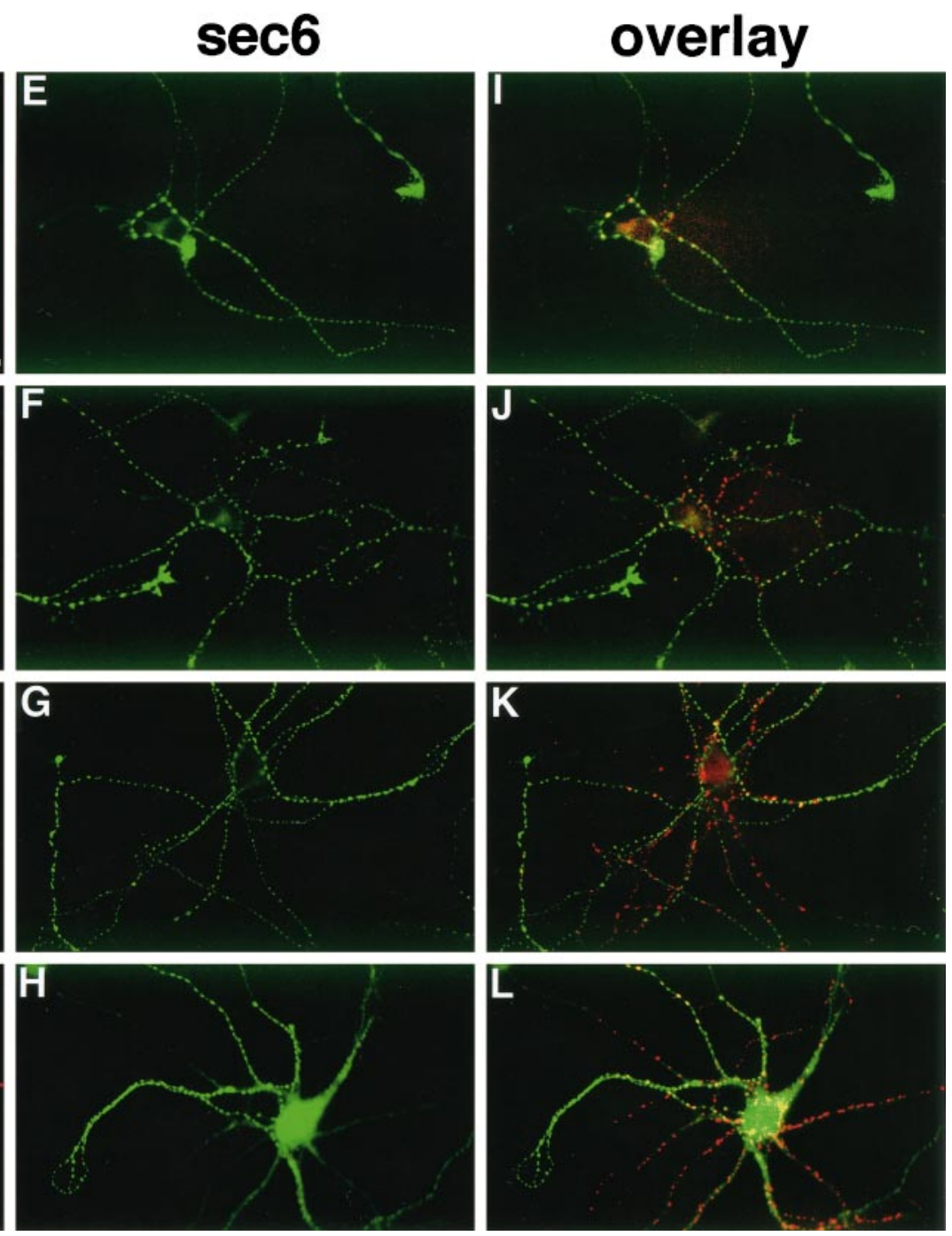

Figure 6. sec6 immunoreactivity precedes the accumulation of synapsin 1 at potential synaptic sites. Time course of sec6 and synapsin 1 immunoreactivity in maturing neurons in culture. Embryonic hippocampal neurons fixed and labeled at $4(A, E, I), 6(B, F, J), 9(C, G, K)$, and $12(D, H, L)$ div. At 4 div, synapsin1 labeling was present in the newly growing axon at sites of contact with dendrites $(A)$. sec6 labeling $(E)$ was present in a periodic distribution along the length of the axon, including the sites that were forming synapses as shown in yellow in the overlay image $(I)$. At 6 div, many more synapses were present around the cell body and proximal dendrites $(B)$. The characteristic labeling of sec6 was present in most axons $(F)$; however, many synapsin1-positive sites were devoid of sec6 $(J)$. At 9 div, synapsin1-positive synapses were found along the cell body and dendrites $(C)$. Many incoming processes, including the growth cone, were labled with sec6 but not synapsin1 antibodies $(G)$. The overlay image demonstrates little overlap of the two fluorescence signals $(K)$. At 12 div, many synapses were observed $(D)$. Diff use labeling of sec6 was observed in the cell body $(H)$. The dendrites were faintly, diffusely labeled. One or two incoming axons were forming connections and demonstrated intense periodic labeling of sec6. The processes contained either synapsin1 or sec6 labeling $(L)$, which was probably because of the different stages of maturity. Scale bar, $28 \mu \mathrm{m}$.

were stimulated with field electrodes in the presence of $\mathrm{Ca}^{2+}$ and FM1-43 and then washed for $20 \mathrm{~min}$ and imaged. Cells were then stimulated in the absence of FM1-43 to unload the endocytosed dye. After imaging, cells were fixed and labeled with antibodies to sec6 and synapsin1, and the same cell fields were imaged for immunofluorescence. The three fluorescence signals were compared for each FM1-43-, sec6-, or synapsin1-positive site.

We quantified the incidence of colocalization between sec6, synapsin1, and FM1-43 (Tables 1, 2). At this intermediate time during synaptogenesis, we expected to observe presynaptic specializations in a variety of stages of maturity. As shown in Figure 8, sites at which FM1-43 was taken up through endocytosis were usually labeled by the synapsin1 antibody $(>82 \%)$. sec6-positive sites were scored for the presence of FM1-43 and/or synapsin1 (Table 1). Sixty-six percent of sec6 sites did not contain either
FM1-43 or synapsin1 (Fig. 8, 1), indicating that most regions containing the sec6/8 complex are not functional synapses. Exocytosis of vesicles may still occur at these synapsin1-negative locations, but these vesicles would not be detected using FM1-43, which only detects locally confined clusters of vesicles undergoing cycles of exocytosis and endocytosis. In fact, only $4 \%$ of sec 6 sites contained FM1-43 in the absence of synapsin1; these likely fall within experimental error of the fixation-immunostaining technique. Fourteen percent of sec6 sites contained synapsin 1 but not FM1-43 (Fig. 8, 2). These may represent newly formed nonfunctional synapses. Sixteen percent of sec6 sites contained both synapsin1 and FM1-43 (Fig. 8, 3), indicating that the sec6/8 complex can be present in functional synapses.

To investigate the significance of the sec6/8 complex in mature synapses, functional synapses were scored for the presence of the 


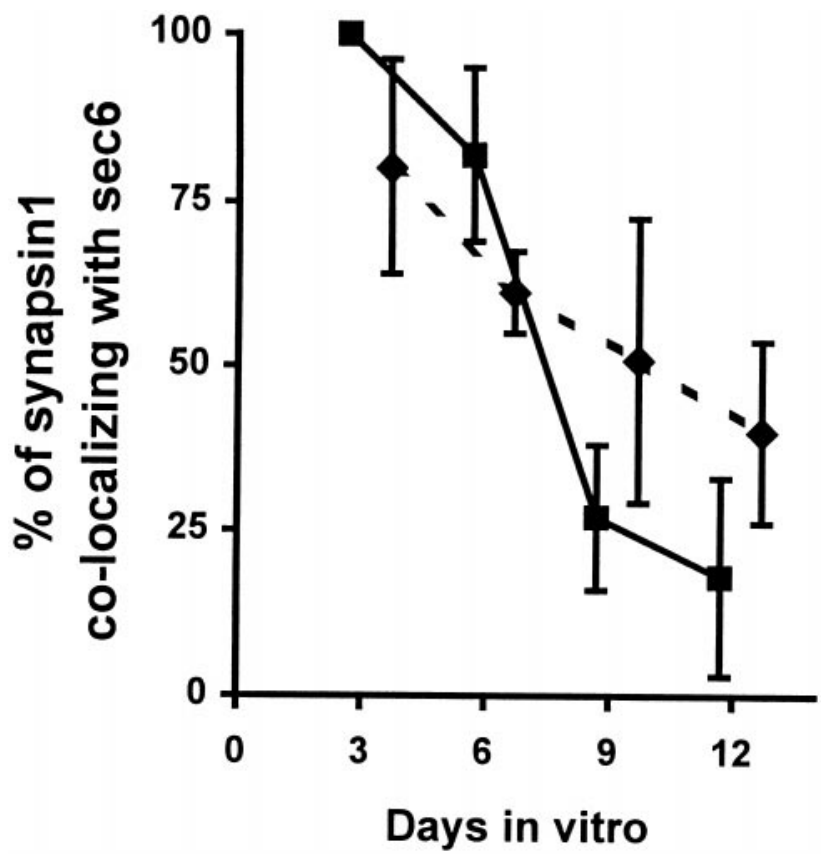

Figure 7. sec6/8 complex and synapsin 1 colocalization changes during development. The number of synapsin 1 sites containing sec6 decreased significantly as the neurons matured. Colocalization was quantified by scoring sec6 and synapsin1 sites manually under low magnification (solid line). Similar results were obtained using an automated method (dashed line; see Materials and Methods). Although the automated method was less precise, it eliminated the possibility of experimental bias.

sec6/8 complex (Table 2). Only $33 \%$ of synapsin1-FM1-43 sites contained sec6 labeling (Fig. 8, 4), confirming that the sec6/8 complex is not required or is modified in functioning synapses. Synapses containing sec6, synapsin1, and FM1-43 may represent immature synapses because the majority of FM1-43/synapsin1 sites do not contain sec6. These results are consistent with the hypothesis that the sec6/8 complex marks axonal locations that have the capacity to become synapses. As sites mature, the sec6/8 complex is no longer required, and vesicles will cycle locally in the absence of the sec6/8 complex.

\section{DISCUSSION}

Previous studies have promoted the hypothesis that the sec6/8 complex plays a role in specifying sites of exocytosis important for polarized secretion in yeast and MDCK cells. Such a role likely extends to neurons, highly polarized cells where targeting mechanisms are crucial for organized membrane trafficking. We have tested this hypothesis by analyzing the distribution of the mammalian sec6/8 complex in neural tissue and cultured neurons during various stages of development of polarity and synaptogenesis. Our results suggest that the sec6/8 complex defines domains of membrane addition and synaptogenesis in growing axons.

Exactly where vesicles fuse with axonal plasma membrane in developing neurons is ambiguous. Conflicting reports have implicated either the cell body or the terminal growth cone as the important site of vesicle addition for axonal growth (Popov et al., 1993; Craig et al., 1995). It is also possible, in the hippocampal culture system, that membrane addition can occur along the axon, as well as in growth cones (Futerman and Banker, 1996). Additionally, Golgi-derived immature synaptic vesicles may fuse at multiple sites along the axon as they travel toward their ultimate
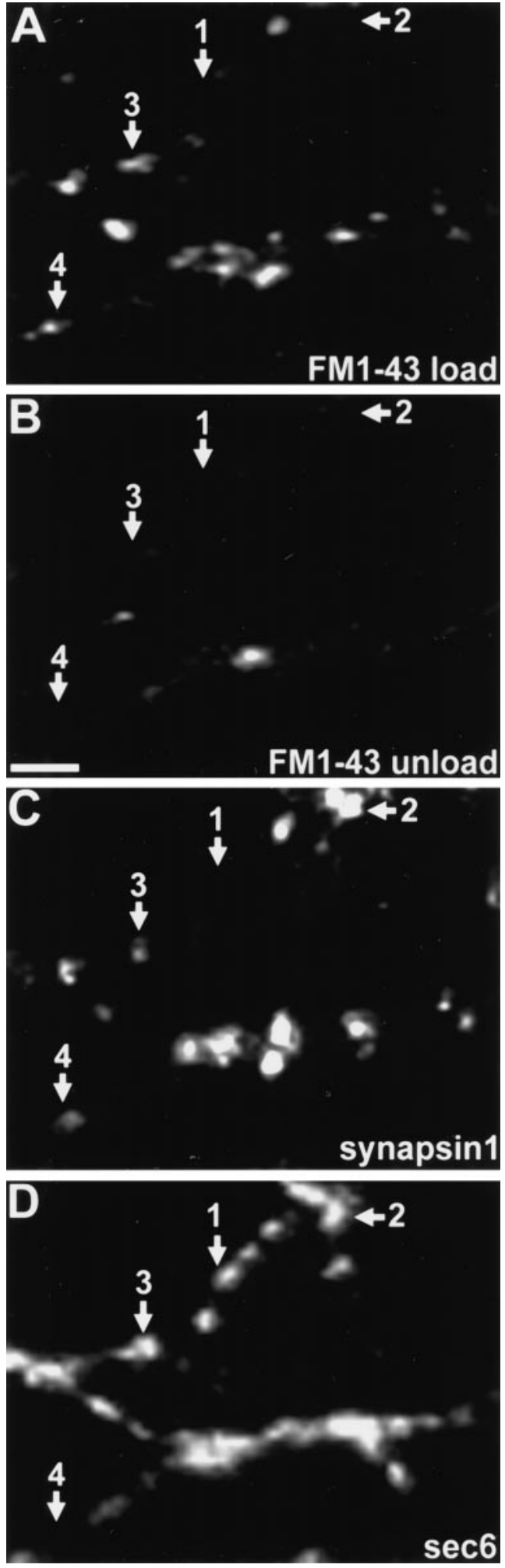

Figure 8. The sec6/8 complex is not required at functional synapses. The presence of actively cycling vesicles was monitored in real time by confocal imaging of neurons loaded with FM1-43. A, An example of processes imaged after stimulation in the presence of FM1-43. $B$, The same field after subsequent stimulation in the absence of FM1-43. $C, D$, The same field after fixation and labeling with antibodies to synapsin $1(C)$ and sec6 $(D) .1$ indicates sec6 sites that contained neither FM1-43 nor synapsin1. 2 indicates sites containing sec6 and synapsin1 but not FM143. 3 indicates sites containing sec6, synapsin1, and FM1-43. 4 indicates sites containing synapsin1 and FM1-43 but not sec6. Scale bar, $3.5 \mu \mathrm{m}$. 

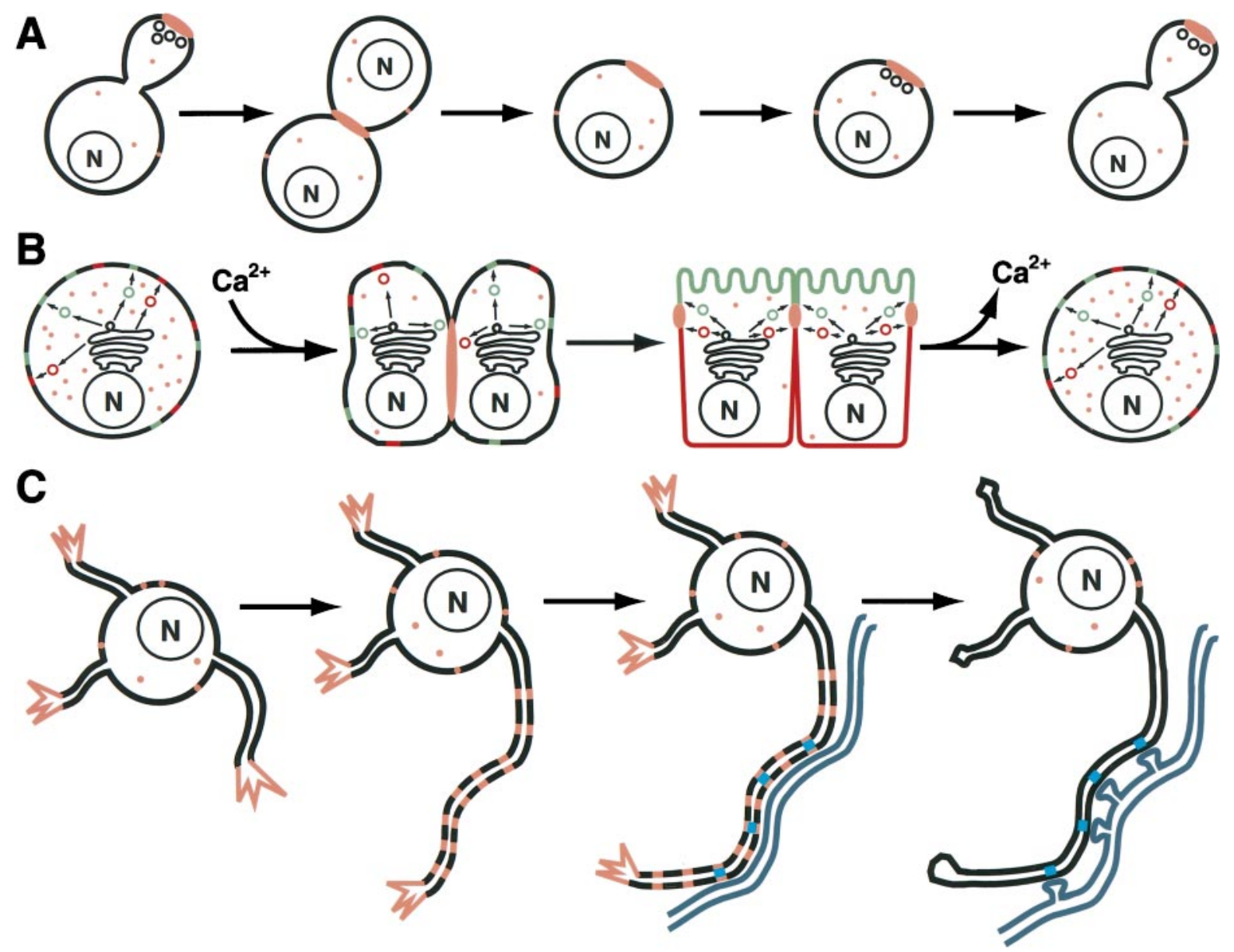

Figure 9. A model of the actions of the sec6/8 complex in vesicle accumulation and synaptogenesis. $A$, A model of the actions of the sec6/8 complex during the life cycle of yeast cells. The sec6/8 complex ( peach) is present at sites of secretion in the tip of the budding daughter cell, at sites of cytokinesis in dividing cells, and in a patch at the bud scar, which designates the site of exocytosis underlying future bud formation (there is a cytosolic pool as well; peach dots) (Finger et al., 1998). $N$, Nucleus. $B$, A model of the role of the sec6/8 complex in polarized secretion in MDCK epithelial cells. Unpolarized MDCK cells target vesicles ( green and red) randomly. The sec6/8 complex ( peach) is dispersed throughout the cytosol. After the addition of Ca ${ }^{2+}$, two cells contact, and the cytoplasmic sec6/8 complex is reorganized to the contacting plasma membrane. Once polarized, the cells target vesicles to either the apical membrane (green) or the basolateral membrane (red). The sec6/8 complex is located along the membrane near the tight junctions, suggesting that the junction is a site to which vesicles are targeted. After depletion of $\mathrm{Ca}^{2+}$, the cells become unpolarized, and the sec6/8 complex is found dispersed in the cytosol (Grindstaff et al., 1998). C, A model of the role of the sec6/8 complex in synaptogenesis. Neurons of increasing maturity are shown from left to right. In young neurons, the sec6/8 complex ( peach) is present in growth cones of neurites. Later, one neurite becomes the axon, leaving the others as dendrites; the sec6/8 complex is organized into periodic domains along the axon. As the neuron matures, vesicle clusters are found in some of the sec6/8 domains (synapsin1-containing vesicle clusters are shown in blue). As synapses are formed between the axon and dendrites (a dendrite is shown in gray), local clusters of synaptic vesicles are stabilized, and the sec6/8 complex is downregulated.

synaptic destination (Nakata et al., 1998). These processes may require machinery for vesicle fusion along the plasma membrane. Thus, it is possible that the sec6/8 complex is required along growing axons to mediate targeted exocytosis of constitutive and synaptic vesicles.

The results presented here provide the first description of a set of proteins that demarcates domains of the neuronal plasma membrane as sites of possible vesicle exocytosis underlying the development of presynaptic specializations. The sec6/8 complex was observed in periodic axonal domains in cultured hippocampal neurons, reminiscent of previously observed periodic synaptic vesicle exocytosis domains. These periodic regions of potential exocytosis in axons that have not contacted target cells have been described in embryonic hippocampal cultures (Matteoli et al., 1992), dorsal root ganglion neurons (Nakata et al., 1998), and in developing cortical neurons in brain (although these fetal neurons may have postsynaptic partners) (Chun and Shatz, 1989). The periodic distribution of the sec6/8 complex may ensure an appropriate nonoverlapping distribution of synapses in adult neurons. Because the arrival of the sec6/8 complex precedes the appearance of synaptic vesicle clusters, we propose that the sec6/8 complex signals the accumulation of components of the presynaptic terminal at predetermined sites along axons. This may be achieved via the targeting of vesicles containing presynaptic plasma membrane proteins. Probably only a fraction of sec6/8 sites become synapses because they did not always colocalize with vesicle markers or functional vesicle cycling. Other molecules may be required for deciding at which sec6/8 sites vesicles will accumulate in the process of synaptogenesis. Perhaps there are also negative signals that prevent such accumulation in areas immediately surrounding the domains demarcated by the sec6/8 complex. Thus, the sec $6 / 8$ complex may be part of a signaling 
pathway in developing axons important for organizing the compartmentalization of components necessary for presynaptic functioning.

The distribution of the sec6/8 complex is interesting in the context of previous studies in hippocampal cultures, which have demonstrated that parts of the presynaptic specialization are organized early, before functional synapses are formed. Axons have been shown to be competent to rapidly form synapses, suggesting that the presynaptic components are organized before contacts with postsynaptic cells are established (Fletcher et al., 1994). This idea is supported by electron microscopy and liveimaging experiments, which have demonstrated the presence of nonsynaptic vesicle clusters in axons. These clustered vesicles can undergo cycling at the plasma membrane in response to activity and can move in bulk throughout the axon in both anterograde and retrograde directions (Matteoli et al., 1992; Kraszewski et al., 1995; Dai and Peng, 1996; Nakata et al., 1998), indicating that vesicles are capable of accumulating in axons in the absence of contacts with other cells but that contact with a dendrite is necessary for stabilization of a vesicle cluster at a synapse (Kraszewski et al., 1995; Dai and Peng, 1996). The distribution of the sec6/8 complex in axons and filopodia suggest that it may be important for clustering mobile vesicle pools at sites of axodendritic contacts.

The data presented here in neurons is redolent of the observations in yeast and MDCK cells in which the sec6/8 complex is located at sites of and required for polarized secretion (TerBush and Novick, 1995; Finger et al., 1998; Grindstaff et al., 1998) (Fig. $9 A, B)$. One obvious difference between the present and previous data is the downregulation observed in differentiated neurons, which is not seen in polarized MDCK cell monolayers. Polarized MDCK cells are not terminally differentiated but, instead, are continually testing their intercellular connections for retention of polarity as evidenced by the rapid turnover of E-cadherin $(5 \mathrm{hr})$ (Shore and Nelson, 1991). A steady supply of the sec6/8 complex is therefore probably required for targeted exocytosis. The epitope recognized by the sec6 antibody may be modified or occluded by other proteins during synapse development. Alternatively, in mature synapses, the sec6/8 complex may not be needed because the large scale recruitment of vesicles to the synapse no longer occurs. Synaptic vesicles in mature synapses may be able to retain their molecular components, with minimal contribution from the cell body. However, the sec6/8 complex may still be required for neuronal function in adult brain, although its immunoreactivity, and probably abundance, is greatly reduced. For example, the sec6/8 complex may be required for the trafficking of vesicles to new synapses and, as a result, may be present in those cells that sprout new axon terminals or are undergoing axonal growth as a mechanism of synaptic plasticity. A low level of the sec6/8 complex may also be required for the constitutive secretion of Golgi-derived vesicles serving homeostatic functions in neurons. Thus, the sec6/8 complex may be important for general secretion mechanisms used by all cells, and it may have been adapted to serve neuron-specific functions.

In the context of these data, we propose a model of the role for the sec6/8 complex in vesicle targeting in developing neurons (Fig. $9 C)$. In very young neurons, the sec $6 / 8$ complex is present in the growth cones of all processes extending from the cell body. The sec6/8 complex is also clustered in periodic regions along the length of the developing axon. Eventually, immature synaptic vesicles and associated proteins (synaptotagmin, synapsin1, etc.) are clustered in some sec6/8 complex-containing domains. Once an axon contacts a dendrite (Ziv and Smith, 1996), the synapse is stabilized, leading to downregulation of the sec6/8 complex. A pool of mature synaptic vesicles is left behind at presynaptic active zones. It is possible that the need for the sec $6 / 8$ complex is obviated by postsynaptic signals. At this stage in culture, the distal tip of the axon may still be growing and forming synapses, thus preserving the need for the sec $6 / 8$ complex in the axon in addition to a possible role in constitutive secretion. Furthermore, a diff use pool of the sec6/8 complex remains in the cell body, the dendrites, and perhaps axons directing constitutive secretion.

An important future topic of research will be the description of the molecular mechanisms underlying the targeting and localization of the sec6/8 complex to potential presynaptic sites. If, as proposed, the sec6/8 complex is involved in establishing potential active zones for synaptic vesicle docking and fusion, it may be possible to use the proteins of the complex as molecular handles with which to characterize other molecules involved in axonal differentiation and synapse formation. The activation of the sec6/8 complex in response to experience in adult brain may result in the formation of new synapses. One prediction is that the sec6/8 complex would rapidly respond to molecules that signal either stabilization or modification of synapses. Such a regulatory switch would, through the sec6/8 complex, redirect membrane flow in nerve terminals from constitutive to local recycling (Xie and Poo, 1986; Zoran et al., 1991). Identification of these sec6/8 complex interacting proteins will provide important insights into the biochemical basis of neural circuitry development and plasticity.

\section{REFERENCES}

Banker GA, Cowan WM (1977) Rat hippocampal neurons in dispersed cell culture. Brain Res 126:397-425.

Bartlett WP, Banker GA (1984) An electron microscopic study of the development of axons and dendrites by hippocampal neurons in culture. II. Synaptic relationships. J Neurosci 4:1954-1965.

Betz WJ, Mao F, Smith CB (1996) Imaging exocytosis and endocytosis. Curr Opin Neurobiol 6:365-371.

Bowser R, Novick P (1991) Sec15 protein, an essential component of the exocytotic apparatus, is associated with the plasma membrane and with a soluble 19.5S particle. J Cell Biol 112:1117-1131.

Bowser R, Muller H, Govindan B, Novick P (1992) Sec8p and Sec15p are components of a plasma membrane-associated $19.5 \mathrm{~S}$ particle that may function downstream of Sec4p to control exocytosis. J Cell Biol 118:1041-1056.

Broadie K, Prokop A, Bellen JJ, O'Kane CJ, Schulze KL, Sweeney ST (1995) Syntaxin and synaptobrevin function downstream of vesicle docking in Drosophila. Neuron 15:663-673.

Calakos N, Scheller RH (1996) Synaptic vesicle biogenesis, docking and fusion: a molecular description. Physiol Rev 76:1-29.

Chun JJM, Shatz CJ (1988) Redistribution of synaptic vesicle antigens is correlated with the disappearance of a transient synaptic zone in the developing cortex. Neuron 1:297-310.

Chun JJM, Shatz CJ (1989) The earliest-generated neurons of the cat cerebral cortex: characterization by MAP2 and neurotransmitter immunohistochemistry during fetal life. J Neurosci 9:1648-1667.

Craig AM, Banker G (1994) Neuronal polarity. Annu Rev Neurosci 17:267-310.

Craig AM, Wyborski RJ, Banker G (1995) Preferential addition of newly synthesized membrane protein at axonal growth cones. Nature 375:592-594.

Dai Z, Peng HB (1996) Dynamics of synaptic vesicles in cultured spinal cord neurons in relationship to synaptogenesis. Mol Cell Neurosci 7:443-452.

Dotti CG, Banker GA (1987) Experimentally induced alteration in the polarity of developing neurons. Nature 330:254-256.

Dotti CG, Sullivan CA, Banker GA (1988) The establishment of polarity by hippocampal neurons in culture. J Neurosci 8:1454-1468.

Drubin DG, Nelson WJ (1996) Origins of cell polarity. Cell 84:335-344. 
Finger FP, Novick P (1997) Sec3p is involved in secretion and morphogenesis in Saccharomyces cerevisiae. Mol Biol Cell 8:647-662.

Finger FP, Hughes TE, Novick P (1998) Sec3p is a spatial landmark for polarized secretion in budding yeast. Cell 92:559-571.

Fletcher TL, Cameron P, De Camilli P, Banker G (1991) The distribution of synapsin1 and synaptophysin in hippocampal neurons developing in culture. J Neurosci 11:1617-1626.

Fletcher TL, De Camilli P, Banker G (1994) Synaptogenesis in hippocampal cultures: evidence indicating that axons and dendrites become competent to form synapses at different stages of neuronal development. J Neurosci 14:6695-6706.

Futerman A, Banker G (1996) The economics of neurite outgrowth-the addition of new membrane to growing axons. Trends Neurosci 19:144-149.

Galli T, Garcia EP, Mudigl O, Chilcote TJ, De Camilli P (1995) v- and t-SNAREs in neuronal exocytosis: a need for additional components to define sites of release. Neuropharmacology 34:1351-1360.

Grindstaff KK, Yeaman C, Anandasabapathy N, Hsu SC, RodriguezBoulan E, Scheller RH, Nelson WJ (1998) Sec6/8 complex is recruited to cell-cell contacts and specifies transport vesicle delivery to the basal-lateral membrane in polarized epithelial cells. Cell 93:731-740.

Guo W, Roth D, Gatti E, De Camilli P, Novick P (1997) Identification and characterization of homologues of the exocyst component Sec10p. FEBS Lett 404:135-139.

Hanson PI, Roth R, Morisaki H, Jahn R, Heuser JE (1997) Structure and conformational changes in NSF and its membrane receptor complexes visualized by quick-freeze/deep-etch electron microscopy. Cell 90:523-535.

Hay JC, Scheller RH (1997) SNAREs and NSF in targeted membrane fusion. Curr Opin Cell Biol 9:505-512.

Hazuka CD, Hsu SC, Scheller RH (1997) Characterization of a subunit of the rat brain rsec6/8 complex. Gene 187:67-73.

Hsu SC, Ting AE, Hazuka CD, Davanger S, Kenny JW, Kee Y, Scheller RH (1996) The mammalian brain rsec6/8 complex. Neuron 17:1209-1219.

Hsu SC, Hazuka CD, Roth R, Foletti DL, Heuser J, Scheller RH (1998) Subunit composition, protein interactions and structures of the mammalian brain sec6/8 complex and septin filaments. Neuron 20:1111-1122.

Jacobsson G, Bean AJ, Scheller RH, Juntti-Berggren L, Deeney JT, Berggren PO, Meister B (1994) Identification of synaptic proteins and their isoform mRNAs in compartments of pancreatic endocrine cells. Proc Natl Acad Sci USA 91:12487-12491.

Kee Y, Yoo JS, Hazuka CD, Peterson KE, Hsu SC, Scheller RH (1997) Subunit structure of the mammalian exocyst complex. Proc Natl Acad Sci USA 94:14438-14443.

Kraszewski K, Mundigl O, Daniell L, Verderio C, Matteoli M, De Camilli P (1995) Synaptic vesicle dynamics in living cultured hippocampal neurons visualized with CY3-conjugated antibodies directed against the lumenal domain of synaptotagmin. J Neurosci 15:4328-4342.

Lin R, Scheller RH (1997) Structural organization of the synaptic exocytosis core complex. Neuron 19:1087-1094.
Malgaroli A, Ting AE, Wendland B, Bergamaschi A, Villa A, Tsien RW, Scheller RH (1995) Presynaptic component of long-term potentiation visualized at individual hippocampal synapses. Science 268:1624-1628.

Matteoli M, Takei K, Perin MS, Sudhof TC, De Camilli P (1992) Exoendocytotic recycling of synaptic vesicles in developing processes of cultured hippocampal neurons. J Cell Biol 117:849-861.

Monde'sert G, Clarke DJ, Reed SI (1997) Identification of genes controlling growth polarity in the budding yeast Saccharomyces cerevisiae: a possible role of $N$-glycosylation and involvement of the exocyst complex. Genetics 147:421-434.

Nakata T, Terada S, Hirokawa N (1998) Visualization of the dynamics of synaptic vesicle and plasma membrane proteins in living axons. J Cell Biol 140:659-674.

Novick P, Field C, Schekman R (1980) Identification of 23 complementation groups required for post-translational events in the yeast secretory pathway. Cell 21:205-215.

Popov S, Brown A, Poo M (1993) Forward plasma membrane flow in growing nerve processes. Science 259:244-246.

Potenza M, Bowser R, Müller H, Novick P (1992) SEC6 encodes an 85 $\mathrm{kDa}$ soluble protein required for exocytosis in yeast. Yeast 8:549-558

Ryan TA, Smith SJ (1995) Vesicle pool mobilization during action potential firing at hippocampal synapses. Neuron 14:983-989.

Ryan TA, Reuter H, Wendland B, Schweizer FE, Tsien RW, Smith SJ (1993) The kinetics of synaptic vesicle recycling measured at single presynaptic boutons. Neuron 11:713-724.

Scheller RH (1995) Membrane trafficking in the presynaptic nerve terminal. Neuron 14:893-897.

Shore EM, Nelson WJ (1991) Biosynthesis of the cell adhesion molecule uvomorulin (E-cadherin) in Madin-Darby canine kidney epithelial cells. J Biol Chem 266:19672-19680.

Sudhof TC (1995) The synaptic vesicle cycle: a cascade of proteinprotein interactions. Nature 375:645-653.

TerBush DR, Novick P (1995) Sec6, Sec8, and Sec15 are components of a multisubunit complex which localizes to small bud tips in Saccharomyces cerevisiae. J Cell Biol 130:299-312.

TerBush DR, Maurice T, Roth D, Novick P (1996) The exocyst is a multiprotein complex required for exocytosis in Saccharomyces cerevisiae. EMBO J 15:6483-6494.

Ting AE, Hazuka CD, Hsu SC, Kirk MD, Bean AJ, Scheller RH (1995) rSec6 and rSec8, mammalian homologs of yeast proteins essential for secretion. Proc Natl Acad Sci USA 92:9613-9617.

Weber T, Zemelman BV, McNew JA, Westermann B, Gmachl M, Parlati F, Sollner TH, Rothman JE (1998) SNAREpins: minimal machinery for membrane fusion. Cell 92:759-772.

Xie XP, Poo MM (1986) Initial events in the formation of neuromuscular synapse: rapid induction of acetylcholine release from embryonic neuron. Proc Natl Acad Sci USA 83:7069-7073.

Ziv NE, Smith SJ (1996) Evidence for a role of dendritic filopodia in synaptogenesis and spine formation. Neuron 17:91-102.

Zoran M, Doyle RT, Haydon PG (1991) Target contact regulates the calcium responsiveness of the secretory machinery during synaptogenesis. Neuron 6:145-151. 